\title{
O controle de autoridade no domínio bibliográfico: os catálogos em livros e em fichas
}

\author{
Fabrício Silva Assumpção \\ Plácida Leopoldina Ventura Amorim da Costa Santos \\ Universidade Estadual Paulista "Júlio de Mesquita Filho" - UNESP, Brasil
}

Zaira Regina Zafalon

Universidade Federal de São Carlos - UFSCar, Brasil

REVIEW

\begin{abstract}
Resumo
Por meio de uma revisão de literatura, este estudo tem por objetivo analisar o controle de autoridade e o papel dos dados de autoridade nos catálogos em livros e em fichas. Considerando a falta de univocidade entre as entidades utilizadas como pontos de acesso nos catálogos (pessoas, entidades coletivas, conceitos, etc.) e os nomes pelos quais essas entidades são conhecidas, é discutido o controle de autoridade e os conceitos a ele relacionados. Em seguida, são descritos os catálogos em livros e em fichas, com ênfase na organização e nos pontos de acesso neles utilizados, e destacadas as principais contribuições de Panizzi, Jewett, Cutter e da American Library Association relacionadas ao controle de autoridade. Por fim, considera-se que os registros de autoridade atuais tiveram suas funções e seus elementos definidos ainda no contexto dos catálogos analógicos, em especial dos catálogos em fichas, de modo que os resultados deste estudo contribuem para a compreensão dos registros e arquivos de autoridade atuais e do próprio conceito de controle de autoridade, assim como para a discussão sobre novos conjuntos de regras de descrição, padrões para o intercâmbio de dados, práticas e tecnologias para a publicação de dados bibliográficos e de autoridade.
\end{abstract}

Palavras-chave

Catalogação descritiva; catálogos bibliográficos; catálogos em fichas; catálogos em livros; controle de autoridade; história da catalogação

\section{Authority control in the bibliographic domain: book and card catalogs}

\begin{abstract}
In this paper we use a literature review to analyze the authority control and the role of authority data in book and card catalogs. Considering the ambiguity in the relation among the entities used as access points in catalogs (persons, corporate bodies, concepts, etc.) and the names by which these entities are known, we discuss authority control and the concepts related to it. Then, we describe book catalogs and card catalogs focusing on their arrangement and access points, and we highlight the main contributions of Panizzi, Jewett, Cutter, and American Library Association related to authority control. As conclusions, we point out that current authority records had their functions and elements defined in analogic catalogs context, especially in card catalogs. The results presented in this paper contribute to understanding of the current authority records and files and of the authority control concept itself, as well as they support the discussion about new descriptive rules, data interchange standards, practices and technologies used in the publication of bibliographic and authority data.
\end{abstract}

\section{Keywords}

Authority control; bibliographic catalogs; book catalogs; card catalogs; descriptive cataloging; history of cataloging 


\section{Introdução}

A partir da segunda metade do século XIX, o desenvolvimento da Biblioteconomia e, já no século XX, da Ciência da Informação, contou com o desenvolvimento da Catalogação, compreendida aqui como o conjunto de conhecimentos acerca das teorias, dos instrumentos de representação e das tecnologias relacionadas à construção e à gestão de catálogos, principalmente no âmbito das bibliotecas.

Os catálogos, enquanto principais resultados tangíveis dos processos descritivos e temáticos da Catalogação realizados nas bibliotecas, são entendidos como ferramentas para o acesso aos recursos informacionais de uma instituição e como meios de comunicação entre os recursos informacionais e seus usuários (MEY; SILVEIRA, 2009, p. 12).

As unidades básicas que compõem os catálogos são denominadas registros bibliográficos. Para a Declaração dos Princípios Internacionais de Catalogação, um registro bibliográfico é "um conjunto de elementos de dados que descreve e provê acesso a um recurso bibliográfico e identifica obras e expressões relacionadas" (INTERNATIONAL FEDERATION OF LIBRARY ASSOCIATIONS AND INSTITUTIONS, 2009, p. 10, tradução nossa). Tradicionalmente, um registro bibliográfico é composto por uma descrição bibliográfica, "um conjunto de dados bibliográficos que identifica um recurso bibliográfico", e por um ou mais pontos de acesso, "um nome, termo, código, etc. por meio do qual dados bibliográficos ou de autoridade são buscados e identificados" (INTERNATIONAL FEDERATION OF LIBRARY ASSOCIATIONS AND INSTITUTIONS, 2009, p. 9, tradução nossa).

Ainda que o catálogo com seus registros bibliográficos seja o principal objeto de estudo da Catalogação, deve ser observado que outros meios de acesso à informação com outros tipos de registros e de dados também são de interesse dessa disciplina. Entre esses meios, têm-se os arquivos de autoridade, que, por meio de dados de autoridade, prestam-se principalmente ao controle dos pontos de acesso utilizados nos catálogos, controle este comumente referido como controle de autoridade.

Este estudo é parte de uma pesquisa de doutorado que tem entre seus objetivos o entendimento das funções dos dados de autoridade nos catálogos. Para esse entendimento, são analisados os três principais tipos de catálogos observados ao longo da história da Catalogação: catálogos em livros, catálogos em fichas e catálogos digitais. Essa análise é conduzida em duas partes. A primeira parte, apresentada neste artigo, tem por objetivo analisar o controle de autoridade e o papel dos dados de autoridade nos catálogos em livros e em fichas. A segunda parte, que se volta aos catálogos digitais, é apresentada no artigo "O controle de autoridade no domínio bibliográfico: os catálogos digitais".

Este estudo, realizado a partir de uma revisão de literatura, justifica-se pela necessidade de compreensão do controle de autoridade nos catálogos, que pode auxiliar (1) no entendimento dos instrumentos de representação atualmente utilizados no domínio bibliográfico, (2) na construção de sistemas para catálogos digitais e (3) na avaliação e (4) na implementação de novos padrões voltados aos dados, registros e arquivos de autoridade.

Os resultados da primeira parte deste estudo são apresentados neste artigo em quatro seções: conceituação de controle de autoridade e dos termos relacionados a ele (seção 2), descrição e análise do controle de autoridade nos catálogos em livros (seção 3), descrição e análise do controle de autoridade nos catálogos em fichas (seção 4) e considerações finais (seção 5).

\section{Controle de autoridade}

Nos catálogos digitais atuais, de modo geral, qualquer dado presente em um registro bibliográfico pode ser utilizado para sua recuperação, por exemplo, a data de publicação ou o idioma do recurso informacional representado pelo registro. Ao longo da história da Catalogação, no entanto, restrições de ordem prática e tecnológica limitaram quais dados seriam utilizados para a recuperação dos registros bibliográficos nos catálogos. Assim, de modo geral, os dados escolhidos foram o título do recurso informacional, os nomes dos responsáveis por ele e os termos referentes aos conceitos tratados em seu conteúdo. 
A utilização desses dados, principalmente dos nomes e dos termos, como eram apresentados nos recursos informacionais, por exemplo, o nome do autor como indicado na página de rosto de um livro ou o termo utilizado pelo autor para se referir a um conceito no decorrer do texto, poderia causar problemas na recuperação dos registros bibliográficos, uma vez que os responsáveis e os conceitos, aqui generalizados sob o termo entidades,1 não mantêm relações de univocidade com seus nomes, visto que:

- uma entidade poderia ser representada por mais de um nome, tanto nos recursos informacionais, quanto no conjunto de conhecimentos do usuário do catálogo, por exemplo: uma pessoa poderia ser representada por seu nome completo, nome abreviado, nome anterior ou posterior ao casamento, etc.; uma entidade coletiva poderia ser representada por seu nome oficial, nome pelo qual é mais conhecida, nome anterior, nome mais recente, sigla, nome em outro idioma ou alfabeto, etc.; um conceito poderia ser representado por um nome científico, popular ou local, uma sigla, por variações lexicais, etc.;

- $\quad$ um nome poderia representar mais de uma entidade, tanto nos recursos informacionais, quanto no conjunto de conhecimentos do usuário do catálogo; por exemplo, um mesmo nome pode ser utilizado por diversas pessoas ou entidades coletivas e um mesmo termo pode se referir a diferentes conceitos em diferentes áreas do conhecimento.

Essas duas situações dificultariam a recuperação dos registros bibliográficos nos catálogos, pois (1) resultariam na recuperação de registros bibliográficos irrelevantes para o usuário, nos casos em que um nome representasse mais de uma entidade, e (2) impediriam a recuperação de todos os registros bibliográficos associados a uma entidade, por exemplo, todos os registros dos livros de um mesmo autor ou sobre um mesmo conceito, nos casos em que uma entidade fosse representada por mais de um nome. A busca por soluções para os problemas de recuperação nos catálogos decorrentes da falta de univocidade entre as entidades e seus nomes levou ao surgimento do conceito de controle de autoridade.

As definições de controle de autoridade encontradas na literatura, mesmo voltando-se para a necessidade de garantir a univocidade entre as entidades e os nomes utilizados para representá-las nos catálogos, apresentam diferentes entendimentos acerca do que se constitui como o controle de autoridade: alguns autores entendem o "controle de autoridade como um estado que se alcança por meio da realização de um conjunto de atividades", enquanto outros entendem o controle de autoridade como "o próprio conjunto de atividades ou algumas atividades desse conjunto" (ASSUMPÇÃO; SANTOS, 2012, p. 5-6).

Para Taylor (1984, p. 2 apud CLACK, 1988, p. 36, tradução nossa), o controle de autoridade é "o processo de manter a consistência dos cabeçalhos 2 em um arquivo bibliográfico por meio de referências a um arquivo de autoridade". Clack (1988, p. 35, tradução nossa) destaca que o controle de autoridade é um "[...] processo técnico executado em um arquivo bibliográfico para prover estrutura a esse arquivo", sendo que esse processo envolve

[...] vários processos interdependentes: (1) a certificação de que o cabeçalho da entrada é único, (2) a aplicação de um conjunto rígido de padrões para a escolha da forma dos cabeçalhos das entradas, e (3) a criação de vínculos entre as entradas autorizadas e todas as variações dessas entradas. (CLACK, 1988, p. 35, tradução nossa).

Além de entender que o controle de autoridade é alcançado com a consistência dos pontos de acesso utilizados em um catálogo, Burger (1985, p. 3, tradução nossa) aponta como necessário ao controle de autoridade o estabelecimento de políticas de catalogação que garantam tal consistência: "O controle de autoridade é alcançado quando os cabeçalhos utilizados em um catálogo estão consistentes e quando um mecanismo (o arquivo de autoridade) e políticas de catalogação relacionadas foram estabelecidos para assegurar essa consistência".

Chan (1994, p. 3) também considera o controle de autoridade algo alcançado em um catálogo quando termos uniformes são utilizados para representar os nomes e os assuntos que servem como pontos de acesso para os registros bibliográficos, de modo que os registros bibliográficos associados a uma mesma entidade não se dispersem entre os sinônimos e as formas variantes dos nomes. Para tanto, a autora entende como atividades 
de controle de autoridade (1) a integração de registros de autoridade padronizados no sistema local e (2) a preparação de registros de autoridade para os nomes e os assuntos não disponíveis nos arquivos de autoridade utilizados como padrão, por exemplo, nos arquivos de autoridade de uma agência bibliográfica nacional ou programa de catalogação cooperativa (CHAN, 1994, p. 13-14). Taylor e Joudrey (2009, p. 249, tradução nossa) definem o controle de autoridade como "[...] o resultado do processo de manutenção da consistência da forma verbal utilizada para representar um ponto de acesso e dos processos adicionais de apresentação dos relacionamentos entre nomes, obras e assuntos".

Sobre a importância do controle de autoridade, Assumpção e Santos (2012), ao analisarem as declarações de Cutter, de Verona, de Domanovszky, de Lubetzky e de Svenonius acerca dos objetivos e funções dos catálogos, assim como aquelas apresentadas nos "Princípios de Paris", no Functional Requirements for Bibliographic Records (FRBR) e na Declaração dos Princípios Internacionais de Catalogação, destacam que o controle de autoridade é necessário para que o catálogo permita ao usuário encontrar um recurso informacional conhecido, encontrar um conjunto de recursos informacionais que compartilham determinada característica e navegar no catálogo por meio dos relacionamentos existentes entre as entidades.

Alguns autores, ao definirem o controle de autoridade o relacionam ao conceito de trabalho de autoridade. Segundo Marais (2004, p. 62, tradução nossa), o "controle de autoridade é um termo abrangente e genérico para os processos técnicos do trabalho de autoridade e objetiva alcançar o controle sobre as formas variantes dos pontos de acesso". Para Burger (1985, p. 3, tradução nossa), o "trabalho de autoridade permite que o controle de autoridade ocorra". O autor, na falta de uma definição abrangente sobre o escopo e o propósito, opta por inferir, a partir da literatura, que o trabalho de autoridade consiste, ao menos, em cinco processos:

1. a criação de registros de autoridade (que, por sua vez, são utilizados para criar registros bibliográficos autorizados);

2. a compilação de registros em um arquivo de autoridade;

3. a vinculação do arquivo de autoridade ao arquivo bibliográfico; juntos eles formam um sistema de autoridade;

4. a manutenção do arquivo e do sistema de autoridade;

5. a avaliação do arquivo e do sistema de autoridade. (BURGER, 1985, p. 3, grifo do autor, tradução nossa).

Assumpção e Santos (2012, p. 6), concordam com Burger e enfatizam que a consistência necessária ao controle de autoridade implica "[...] uma entidade ser identificada por apenas um ponto de acesso autorizado e um ponto de acesso autorizado identificar somente uma entidade", o que é destacado por Chan (2007, p. 164-165) com o princípio dos cabeçalhos uniformes e o princípio dos cabeçalhos únicos.

Maxwell (2002, p. 3-4, tradução nossa) ressalta a importância do trabalho de autoridade enquanto responsável por manter registros das decisões tomadas em relação a cada ponto acesso autorizado utilizado no catálogo. Os registros aos quais o autor se refere são hoje denominados registros de autoridade. Segundo a Declaração dos Princípios Internacionais de Catalogação, um registro de autoridade é "um conjunto de elementos de dados que identifica uma entidade e pode ser utilizado para facilitar o acesso ao ponto de acesso autorizado para a entidade ou a exibição de qualquer ponto de acesso para ela" (INTERNATIONAL FEDERATION OF LIBRARY ASSOCIATIONS AND INSTITUTIONS, 2009, p. 9, tradução nossa).

Os registros de autoridade, quando reunidos, constituem os arquivos de autoridade (BURGER, 1985, p. 3; TAYLOR, 2006, p. 19-20). Calhoun (1998, tradução nossa) lista quatro funções dos arquivos de autoridade: (1) "função de autoridade", relativa à manutenção da consistência nos catálogos; (2) "função de localização", relativa à habilidade dos usuários buscarem e encontrarem entidades utilizando um de seus vários nomes; (3) "função informativa", relativa à documentação das fontes de informação consultadas pelo catalogador para a 
criação dos pontos de acesso; e (4) "função de manutenção", relativa à possibilidade de uso do arquivo de autoridade para a identificação e a correção de erros no catálogo.

O trabalho envolvido no controle de autoridade, por demandar pessoal especializado e tempo, é custoso (MAXWELL, 2002, p. 6; MARAIS, 2004, p. 70), o que faz com que, frequentemente, a busca por palavras-chave não-controladas (keyword searching) seja considerada suficiente para a recuperação de recursos informacionais (TAYLOR; JOUDREY, 2009, p. 189). Nesse sentido, em defesa do controle de autoridade, Taylor e Joudrey (2009, p. 189, tradução nossa) destacam que

[...] pesquisas têm demonstrado que a busca por palavras-chave pode gerar resultados falsos (ou seja, recuperar resultados irrelevantes) porque a palavra recuperada possui um significado diferente do pretendido e pode também reduzir a revocação, pois os sinônimos e os quasesinônimos não foram recuperados com a palavra pesquisada.

A necessidade do controle dos pontos de acesso nos catálogos e a existência de instrumentos para esse controle podem ser observadas no decorrer da história da Catalogação, ora de modo implícito, ora de modo explícito. Como pontuam Alves e Santos (2013, p. 23-24), "[...] as bibliotecas procuraram melhorar, cada vez mais, as formas de construção dos catálogos com o desenvolvimento e a utilização de métodos eficazes e econômicos, sempre utilizando as tecnologias disponíveis." A busca por essa melhoria e o uso constante de tecnologias fizeram com que os catálogos evoluíssem dos tabletes de argila até os bancos de dados bibliográficos atuais, passando, entre outros, pelos catálogos em livros, pelos catálogos em fichas e pelas fitas magnéticas e formatos de intercâmbio de dados desenvolvidos ainda nas décadas de 1960 e 1970. Evoluindo com os catálogos esteve a necessidade de controle dos pontos de acesso e os instrumentos utilizados para tanto, como será apresentado nas seções seguintes, que abordam o controle de autoridade nos catálogos em livros (seção 3) e em fichas (seção 4).

\section{Controle de autoridade nos catálogos em livros}

A utilização de catálogos em livros, constituídos por folhas contendo representações de recursos informacionais e encadernadas no formato de códice, pode ser observada já na Idade Média, quando eram criadas listas dos livros presentes nas bibliotecas dos monastérios. No entanto, as considerações acerca do controle de autoridade nesses tipos de catálogos são traçadas neste estudo a partir, aproximadamente, do século XIX.

Essa delimitação respalda-se em dois principais motivos. Primeiramente, nota-se a escassez de informações sobre os catálogos anteriores a esse período, escassez esta decorrente da falta de acesso às fontes de informação primárias (por exemplo, os catálogos e as regras de catalogação anteriores ao século XIX), e das revisões de literatura e pesquisas históricas que não proveem informações suficientes para uma análise sobre os dados presentes nestes catálogos. Em segundo lugar, tem-se que a partir da segunda metade do século XIX é que tiveram início "as tentativas de consolidação de princípios e de regras unificadas para a representação, no intuito de criar um embasamento teórico para as práticas de descrição” (ALVES; SANTOS, 2013, p. 27).

Segundo Obille (2012, p. 7), a partir da segunda metade do século XIX surgiram objetivos que se tornaram subjacentes aos catálogos e aos instrumentos utilizados em sua construção. Esses objetivos, criados a partir de questões de natureza teórica e não apenas prática, são considerados parte do paradigma vigente ainda hoje na Catalogação (OBILLE, 2012, p. 7).

Desse período remontam os esforços de Panizzi, que, no British Museum, advogou a favor de um compêndio de regras para a elaboração do catálogo da biblioteca do referido museu (LUBETZKY, 2000; SANTOS; PEREIRA, 2014, p. 24-25). Esse compêndio de regras, publicado em 1841 no início do primeiro volume do Catalogue of Printed Books in the British Museum, tornou-se conhecido como as "91 regras de Panizzi" (PANIZZI, 1841) e é considerado um dos primeiros códigos de catalogação de que se tem conhecimento (BARBOSA, 1978, p. 26; GARRIDO ARILLA, 1996, p. 76).

Segundo Lubetzky (2000, p. 425, tradução nossa), o estabelecimento das 91 regras de Panizzi foi parte do primeiro acontecimento pivô da história da catalogação anglo-americana, uma vez que ele 
Diz respeito principalmente ao fundamento ideológico do catálogo - o problema fundamental de quais seriam os objetivos aos quais o catálogo de uma biblioteca deveria ser projetado para servir e os métodos para alcançar esses objetivos. E influenciou as discussões e o curso da catalogação anglo-americana desde então [...].

Na época de Panizzi, os registros dos catálogos eram escritos à mão, datilografados ou impressos (CHAN, 2007, p. 22) e apresentados principalmente em fichas e em páginas agrupadas no formato de códices, os chamados catálogos em livro (SVENONIUS, 2000, p. 62; TILLETT, 1992, p. 24). Nesses catálogos, o modo com que as entradas3 eram arranjadas determinava como elas seriam recuperadas e apresentadas (CHAN, 2007, p. 24).

A tecnologia utilizada na criação dos catálogos em livros - o códice - influenciou diretamente as 91 regras de Panizzi, como destaca Tillett (1989, p. 152, tradução nossa):

Como resultado das restrições econômicas sobre o tamanho dos catálogos em livro e o extenso tempo envolvido na redação de fichas para catálogos escritos a mão, as regras de Panizzi instruíram para que um item bibliográfico fosse descrito completamente uma única vez por meio de uma "entrada".

Garrido Arilla (1996, p. 76) aponta que as 91 regras foram redigidas para a criação de catálogos alfabéticos, principalmente de autores pessoais ou corporativos, que permitissem aos usuários a localização rápida e fácil de uma obra ou o agrupamento das obras de um mesmo autor com suas distintas edições e traduções. A organização das entradas nos catálogos em livros de acordo com as 91 regras é descrita por Svenonius (2000, p. 62, tradução nossa):

Abaixo do nome de cada autor eram listadas, por título, as obras escritas por ele. A primeira edição de uma obra custodiada pela biblioteca era descrita por completo. Se a biblioteca possuísse uma segunda edição, sua entrada seria incluída abaixo da entrada da primeira edição assim: "—_ Another edition." Se necessárias, informações que serviam para distinguir a segunda edição da primeira seriam providas. Se a biblioteca possuísse mais de uma cópia da edição, era descrito: “ Another copy.".

Para a autora, essa forma de organização das entradas

[...] assegurava que cada documento seria integrado ao catálogo no sentido de que seria apresentado no contexto de todas as edições, traduções e adaptações da obra manifestada nele. [...] A exibição hierárquica não só assegurava a integração; ela também alcançava economia de expressão ao limitar o que tinha de ser dito sobre uma entrada a somente aquelas características que não poderiam ser derivadas usando relacionamentos de participação e de inclusão. (SVENONIUS, 2000, p. 62, tradução nossa).

Tillett (1989, p. 152) destaca que Panizzi incluiu remissivas para prover acesso mais completo às entradas e para tornar o catálogo mais que uma mera lista ou inventário da coleção. Sobre a função das remissivas, Svenonius destaca que elas indicavam os relacionamentos não hierárquicos entre os recursos informacionais:

Nos catálogos em livros, os relacionamentos de um tipo não hierárquico (ou seja, outros que não fossem de participação ou de inclusão) eram indicados por remissivas. Remissivas Ver vinculavam obras relacionadas, tal como a Bíblia e um comentário sobre ela. Elas eram usadas também para alcançar a disposição de obras quando o acesso era através de atributos secundários. Por exemplo, se um documento fosse de autoria de duas pessoas, a descrição principal (ou entrada principal) seria feita para o primeiro autor, e sob o nome do autor secundário uma remissiva Ver direcionaria o usuário para a descrição principal, que exibiria o documento em seu contexto integrado. (SVENONIUS, 2000, p. 62-63, tradução nossa).

Svenonius (2000, p. 62-63, tradução nossa) complementa que "a necessidade de remissivas bibliográficas de um tipo não hierárquico para auxiliar na navegação e na disposição foi bem entendida por Panizzi, que observou que quanto maior a quantidade de remissivas, mais útil o catálogo seria". 
Em suas 91 regras, Panizzi especificou o uso de três tipos de remissivas: de nome para nome, de nome para obra e de obra para obra. Tillett (1989, p. 152; 1992, p. 24) explica esses três tipos de remissivas:

- nome para nome: direcionava de uma forma variante do nome para a forma do nome escolhida para a entrada

- $\quad$ nome para obra: direcionava para a entrada de uma obra a partir de cabeçalhos que poderiam ser considerados tão importantes quanto o cabeçalho presente na entrada da obra; esses cabeçalhos que direcionavam para a entrada da obra incluíam nomes pessoais, de entidades coletivas, de conferências, de localizações geográficas e de obras (títulos);

- obra para obra: direcionava de uma obra para outra, comumente de partes de uma obra para a obra como um todo.

Enquanto Panizzi publicava suas regras na Inglaterra, Jewett iniciou nos Estados Unidos a elaboração de um catálogo para o acervo da University of Brown. Esse catálogo foi publicado em 1843 dividido em duas partes: "um catálogo descritivo de todas as obras que a Biblioteca possui e um índice de assuntos" (JEWETT, 1843, p. xix, tradução nossa). Na primeira parte (o "catálogo descritivo"), entradas contendo descrições completas das obras estavam organizadas, de modo geral, pelos nomes dos autores e em ordem alfabética, enquanto que na segunda parte entradas contendo descrições abreviadas com referências às entradas da primeira parte estavam organizadas por assunto também em ordem alfabética (JEWETT, 1843; BIAGETTI, 2008, p. 135). Nas duas partes pode ser observada a presença de remissivas "Ver" (See) e "Ver também" (See also), por exemplo:

- "FOXTON, THOMAS. See BURNET, THOMAS"

- "Holy War. See Crusades"

- $\quad$ “JEBB, JOHN, D. D., B’p of Limerick. Correspondence with Knox. (See Forster Charles) Note. See also BURNET, GILBERT.”

- “LONDON AND WESTMINSTER REVIEW [...] (See also Westminster Review.)"

- "Agriculture [...] See also Gardening" (BROWN UNIVERSITY, 1843, p. 169, 517, 236, $266,491)$.

Segundo Santos e Pereira (2014, p. 26), o catálogo de Jewett "[...] foi elogiado como um avanço na prática catalográfica". Para Biagetti (2008, p. 135, tradução nossa), "[...] o elemento inovador foi o fato de que os cabeçalhos eram apresentados em uma única ordem alfabética, com uma relevante presença de remissivas".

As remissivas de nome para nome utilizadas por Panizzi, junto das remissivas utilizadas por Jewett, podem ser consideradas um dos primeiros meios de se alcançar o que hoje se entende por controle de autoridade, ainda que não seja identificada naquela época a presença de registros de autoridade. Essas remissivas, como concebidas por Panizzi e Jewett, são utilizadas ainda hoje, às vezes sob os nomes ponto de acesso variante, ponto de acesso não autorizado, cabeçalho não autorizado, etc.

\section{Controle de autoridade nos catálogos em fichas}

Ainda no século XIX, o desenvolvimento da Catalogação contou com a colaboração de Cutter que, em 1861, começou a trabalhar com Ezra Abbot na Harvard College Library na construção de "um catálogo público em cartões, planejado para ajudar os leitores no uso da biblioteca” (SANTOS; PEREIRA, 2014, p. 29).

Diferente da maioria dos catálogos de biblioteca contemporâneos que eram na forma de volumes publicados, Abbott e Cutter utilizaram cartões de fichários [index cards, do original em inglês], permitindo, assim, a flexibilidade para acrescentar ou remover itens à vontade, ao invés de ter de esperar até a próxima edição do catálogo. (STROMGREN, 2004, tradução nossa). 
Para Santos e Pereira (2014, p. 29), "o catálogo em cartões de duas polegadas, escritos à mão e armazenados em gavetas etiquetadas, provou ser uma maneira nova e facilitadora para o uso público." Segundo as autoras, em 1868 Cutter assumiu a posição de bibliotecário do Boston Athenaeum, onde trabalhou por 24 anos. Em sua primeira tarefa, a publicação de um catálogo da coleção, Cutter tornou-se pioneiro no uso de cabeçalhos de assunto. O catálogo foi publicado em cinco volumes entre 1874 e 1882, tendo servido como modelo de catálogo dicionário em forma de livro (SANTOS; PEREIRA, 2014, p. 29).

Além de criar um sistema de classificação, a Expansive Classification, e um sistema para a notação de autores, Cutter contribui com a Catalogação também com seu conjunto de regras publicado em 1876 sob o título Rules for a Printed Dictionary Catalogue e que teve sua quarta e última edição em 1904 sob o título Rules for a Dictionary Catalog (CUTTER, 1904). Nessa obra, Cutter apresentou quais seriam as funções dos catálogos, sendo considerado o primeiro autor a definir explicitamente tais funções:

1. Possibilitar que uma pessoa encontre um livro do qual
(A) o autor
(B) o título
é conhecido.
(C) o assunto
2. Apresentar o que a biblioteca possui
(D) de um determinado autor
(E) sobre um determinado assunto
(F) em um determinado tipo de literatura.
3. Auxiliar na escolha de um livro
(G) quanto a sua edição (bibliograficamente).
(H) quanto ao seu caráter (literário ou temático). (CUTTER, 1904, p. 12, tradução nossa).

Para o alcance desses objetivos, Cutter listou seis meios, dos quais três dependiam do uso de remissivas:
1. Entrada por autor com as remissivas necessárias (para A e D).
2. Entrada por título ou remissiva de título (para B).
3. Entrada por assunto, remissivas e tabela de classes de assuntos (para C e E).
4. Entrada de forma e entrada de idioma (para F).
5. Informando a edição e a imprenta, com notas quando necessário (para G).
6. Notas (para H). (CUTTER, 1904, p. 12, tradução nossa).

Com o crescimento dos acervos das bibliotecas surgia a necessidade de inclusão de novas entradas nos catálogos. Nos catálogos em livros, as novas entradas eram acrescentadas nos espaços deixados entre as entradas existentes, preservando, assim, a ordenação já estabelecida. No entanto, conforme esses espaços eram ocupados, novas folhas eram intercaladas no catálogo ou as novas entradas eram acrescentadas fora da ordenação, o que causava dificuldades no uso do catálogo (GUERRINI, 2009, p. 1; SVENONIUS, 2000, p. 63). Uma alternativa seria a impressão de uma nova edição do catálogo incluindo as novas entradas, no entanto, além de custosa, tal alternativa representaria uma demora na atualização do catálogo. 
Nos catálogos em fichas, as entradas eram registradas individualmente4 em papéis retangulares (fichas) (SVENONIUS, 2000, p. 63), que eram ordenados e, então, armazenados em gavetas. Uma vez que as fichas não estavam juntas umas as outras, como ocorria com as folhas de um catálogo em livro, elas poderiam ser movimentadas para permitir a inclusão e a remoção de entradas sem prejuízos à ordenação do catálogo. Com isso, os catálogos em fichas, por sua economia e dinamicidade (FRÍAS; PÉREZ ALVAREZ, 1995, p. 283), passaram a substituir gradualmente os catálogos em livros, de produção custosa e que rapidamente se tornavam desatualizados (TAYLOR, 2006, p. 9).

Ainda que remontem ao século XVIII, quando Abbeé Rosier utilizou fichas na catalogação das publicações da Académie des Sciences, na França, em 1775 (SAYERS; STEWART, 1913, p. 8), os catálogos em fichas popularizaram-se entre as bibliotecas a partir do final do século XIX (CHAN, 2007, p. 22-23). O uso de catálogos em fichas como substitutos dos catálogos em livros deu-se também no Brasil, como descreve Bettencourt (2014) em seu estudo sobre a representação da informação na Biblioteca Nacional. Segundo a autora, catálogos em livros eram utilizados para a representação de coleções de recursos informacionais na Biblioteca Nacional e há evidências do uso de catálogos em fichas a partir de 1876, na época denominados catálogos em cartões (BETTENCOURT, 2014, p. 110).

As primeiras fichas manuscritas eram denominadas "cartões alfabéticos" estavam acompanhadas das respectivas "referências e remissões", todas ordenadas em um "catálogo móvel", disponível para os usuários na chamada sala de consulta pública. (BETTENCOURT, 2014, p. 111).

Além das vantagens mencionadas anteriormente, um dos fatores que contribuíram para a popularização dos catálogos em fichas foi o início do serviço de distribuição de fichas catalográficas em janeiro de 1901 pela Library of Congress (LC), nos Estados Unidos (BARBOSA, 1978, p. 72; SVENONIUS, 2000, p. 63; TILLETT, 1989, p. 154).

A utilização de fichas, por facilitar a reprodução das entradas, alterou o modo com que os componentes dos catálogos eram dispostos e apresentados aos usuários e alavancou o surgimento de outros componentes, tais como as entradas secundárias (TILLETT, 1989, p. 154), que possibilitaram o oferecimento de uma quantidade maior de pontos de acesso aos usuários.

Em um catálogo em livro, de modo geral, a descrição completa de um recurso informacional era apresentada apenas na entrada que iniciava com o ponto de acesso autorizado do responsável principal pelo recurso. Ao buscar pelo nome de um responsável que não fosse o responsável principal, por exemplo, o segundo autor, o usuário poderia encontrar uma descrição abreviada do recurso sob o nome do responsável buscado; essa descrição abreviada funcionava como uma remissiva, pois direcionava o usuário para a entrada na qual a descrição completa do recurso informacional estava presente. Situações semelhantes poderiam ocorrer também com partes de obras, como mencionado anteriormente nos tipos de remissivas definidos nas 91 regras de Panizzi, e com assuntos, como pode ser notado na segunda parte do catálogo de Jewett.

Nos catálogos em fichas, a ficha que iniciava com o ponto de acesso autorizado para o responsável principal era denominada entrada principal. Para prover pontos de acesso adicionais referentes aos demais responsáveis, aos títulos e aos assuntos do recurso informacional, a entrada principal era reproduzida e, no topo de cada cópia, era acrescentado o cabeçalho referente a um responsável, a um título ou a um assunto. As entradas criadas a partir da reprodução da entrada principal passaram a ser denominadas entradas secundárias. Com o surgimento das entradas secundárias, as remissivas contendo descrições abreviadas que apontavam para a descrição completa deixaram de ser utilizadas; as remissivas passaram a ser utilizadas, então, apenas para indicar ao usuário os cabeçalhos autorizados e os cabeçalhos relacionados (TILLETT, 1989, 1992). Cabe notar, no entanto, que o uso de entradas secundárias teve início no século XX de forma gradual e com pouca clareza por parte dos códigos de catalogação, como indica a análise da realizada por Tillett (1989, p. 154-156).

Os catálogos em fichas, por permitirem uma fácil intercalação das entradas, favoreceram a criação dos catálogos dicionários. Nesses tipos de catálogos, todas as entradas, independentemente do tipo do cabeçalho utilizado (responsáveis, títulos ou assuntos), eram reunidas e ordenadas em uma única sequência, de modo que os usuários do catálogo poderiam encontrar, em uma mesma gaveta, entradas com cabeçalhos representando os autores, os títulos e os assuntos dos recursos informacionais (GARRIDO ARILLA, 1996, p. 81; MEY; SILVEIRA, 2009, p. 188). 
Na história da Biblioteca Nacional, Bettencourt (2014, p. 111) descreve o uso inicial de catálogos separados por tipos de pontos de acesso e, posteriormente, a reunião destes catálogos em um catálogo dicionário:

Quanto à sua tipologia, os catálogos em fichas inicialmente representavam os autores principais e os assuntos em módulos separados. A busca poderia ser feita no catálogo de autores ou no catálogo de assuntos. Em seguida, os catálogos passaram a ser enriquecidos com outros pontos de acesso, como título, coleção e todos esses pontos de acesso incluídos em um mesmo módulo, o chamado catálogo dicionário.

Segundo Taylor e Joudrey (2009, p. 51), essa foi a forma padrão de organização das entradas dos catálogos na primeira metade do século XX, no entanto, com o crescimento dos acervos e, consequentemente, dos catálogos, ela tornou-se ineficiente, o que fez com que as bibliotecas dividissem as entradas por tipo de cabeçalho e as mantivessem em catálogos diferentes, por exemplo, um catálogo com os títulos, outro com os assuntos, outro com os responsáveis (LUBETZKY, 1940; TAYLOR, 2006, p. 13, 16).

A partir dos catálogos em fichas é possível observar o surgimento dos registros e arquivos de autoridade. As funções dos catálogos listadas por Cutter já demandavam a existência do controle de autoridade, uma vez que encontrar um recurso informacional conhecido ou um conjunto de recursos informacionais que compartilham determinada característica requer o controle dos pontos de acesso (ASSUMPÇÃO; SANTOS, 2012). Os meios listados por Cutter para o alcance dessas funções explicitam essa necessidade ao incluírem o uso de remissivas para nomes, títulos e assuntos.

Auld (1982, p. 320) aponta que Cutter, em suas regras de catalogação (CUTTER, 1904), reconheceu a necessidade do controle de autoridade por meio da criação de listas de autores do catalogador (cataloger's author list, no original, em inglês). Ainda que com o objetivo de evitar a duplicação de trabalho e não necessariamente de servir de apoio ao uso do catálogo, essas listas assemelhavam-se aos arquivos de autoridade atuais, contemplando elementos hoje presentes nos registros de autoridade: "A lista de autores do catalogador, mantida alfabeticamente, evita a duplicação de trabalho. Ela é um registro da forma do nome (na íntegra) adotada, com uma nota das fontes autorizadas consultadas e das variações do nome." (CUTTER, 1904, p. 133, tradução nossa).

Em 1908, a American Library Association (ALA) publicou a primeira edição de seu código de catalogação, Catalog Rules: Author and Title Entries, que, influenciado pelas regras de Cutter, prescrevia o uso de remissivas, embora não descrevesse o uso de registros de autoridade ou de algo semelhante para manutenção dos cabeçalhos adotados e das remissivas criadas para eles (AULD, 1982, p. 320).

A segunda edição preliminar americana do código da ALA, publicada em 1941 com o título A.L.A. Catalog Rules: Author and Title Entries, incluiu um apêndice de três páginas sobre o uso e a criação de fichas de autoridade (authority cards) (AMERICAN LIBRARY ASSOCIATION, 1941, p. 339-331; AULD, 1982, p. 320). Nesse apêndice, observa-se a reiteração de elementos já listados por Cutter para inclusão nas listas de autores, e a inclusão de novos elementos que aproximam as fichas de autoridade deste código aos registros de autoridade existentes hoje. Alguns desses elementos podem ser observados nesta instrução:

Faça uma ficha de autoridade para cada cabeçalho estabelecido informando o cabeçalho exatamente como ele aparece no catálogo. Liste as fontes consultadas na determinação do cabeçalho indicando aquelas nas quais datas foram encontradas e anotando quaisquer variações no nome ou nas datas encontradas em diferentes fontes. Informe de modo mais breve possível o autor, o título e a data do livro para o qual o cabeçalho foi feito. Indique as remissivas. (AMERICAN LIBRARY ASSOCIATION, 1941, p. 339, tradução nossa).

Além dos nomes pessoais, o apêndice contemplava também exemplos de fichas de autoridade para nomes de entidades coletivas e títulos uniformes. A Figura 1 exemplifica uma ficha de autoridade criada de acordo com as instruções deste apêndice para um ponto de acesso de nome pessoal. 
Lewis, Denslow, 1856-1913.

[Full name: William Herbert Denslow Lewis] see $x$ Lewis, William Herbert Denslow.

\author{
Wich. U. Grad. (full name, d. 1913) \\ vamer. med. direct. 1909 (b. 1856) \\ Jt. auth.: Anderson, T.R. \\ Medical notes ... 1901.
}

Figura 1 - Ficha de autoridade para um nome pessoal

Fonte: American Library Association (1941, p. 339)

No cenário brasileiro, Bettencourt (2014, p. 111) destaca que na Biblioteca Nacional, "Com o objetivo de otimizar a padronização das entradas de autores e de assuntos, foram criados internamente, para uso dos bibliotecários, os catálogos de autoridades de nomes, de assuntos e de editores." Ainda segundo a autora, após a consultoria realizada em 1945 por especialistas dos EUA,

Foram criados catálogos de autoridades de nomes e de assuntos para uso dos catalogadores e classificadores da instituição. O catálogo de autoridades de nomes passou a ser estruturado de acordo com o código de catalogação da ALA, constituindo-se em instrumento auxiliar para a padronização das entradas de nomes pessoais, entidades coletivas e eventos. O catálogo de assuntos seguia o modelo da lista de cabeçalhos de assuntos da Library of Congress. (BETTENCOURT, 2014, p. 117).

Sobre a primeira metade do século XX, Fiuza (1980, p. 143) destaca que neste período

[...] os bibliotecários passaram a sofisticar cada vez mais o catálogo. Os catálogos de identidade [i.e., arquivos de autoridade] se avolumaram, pois de cada nome de autor era feita uma pesquisa minuciosa nas fontes de referência; a catalogação descritiva passou a demandar decisões seríssimas e a quantidade de livros nos depósitos, esperando processamento técnico, se tornou assustadora.

A segunda edição do código da ALA, publicada em 1949, sob o título A.L.A. Cataloging Rules for Author and Title Entries, retomou a abordagem da primeira edição de 1908: apresentava regras sobre remissivas, porém sem contemplar a criação de registros de autoridade. Essa abordagem foi seguida também na criação da primeira e da segunda edição do Anglo-American Cataloguing Rules (AACR), publicadas, respectivamente, em 1967 e 1978 (AULD, 1982, p. 321).

No contexto anglo-americano da catalogação, do qual derivam as práticas de catalogação adotadas hoje no Brasil, a falta de prescrição, por parte dos códigos de catalogação, levou à adoção de convenções para a criação de registros de autoridade nos catálogos em ficha, como destaca Burger (1985, p. 23-24, tradução nossa):

Não há nenhum método ou formato nacionalmente prescrito para a transcrição de dados de autoridade em fichas, mas várias convenções são amplamente utilizadas. A primeira e mais importante convenção é a localização do cabeçalho autorizado no topo da ficha, na mesma forma que ele aparece nas fichas catalográficas. Abaixo dele estão localizadas as remissivas. As remissivas Ver [see from] são indicadas pelo prefixo x e as remissivas Ver também [see also from] são indicadas pelo prefixo xx. Abaixo delas são dadas quaisquer notas. Finalmente, são dados o nome ou as iniciais do catalogador, a data em que registro foi preparado e o número de chamada ou o título da publicação que levou à preparação da ficha de autoridade.

Cabe aqui notar que as edições do código da ALA e do AACR não incluíam regras referentes aos pontos de acesso que representavam o conteúdo dos recursos informacionais, ou seja, os pontos de acesso de assunto, eram tratados apenas os pontos de acesso representando os títulos relacionados aos recursos (título principal, título uniforme, título da série, etc.) e os responsáveis por eles. 
Para os pontos de acesso de assunto, outros instrumentos que não códigos de catalogação passaram a ser utilizados, tais como as listas de cabeçalhos de assunto e, posteriormente, os tesauros. Um exemplo destas listas é Library of Congress Subject Headings (LCSH) iniciada em 1898 e publicada pela primeira vez como uma lista em 1909 (SVENONIUS, 2000, p. 178). Esses instrumentos, com outros como os sistemas de classificação bibliográfica, desenvolveram-se como parte do tratamento temático da informação sob o termo genérico linguagens documentais, recebendo aportes de áreas como a Linguística e a Estatística (CINTRA et al., 1994, p. 33) e desempenhando, além das funções dos arquivos de autoridade de nomes e títulos, outras funções, como a de "organizar o campo conceitual da representação documentária" (DODEBEI, 2014, p. 58).

Ainda que com funções e aportes teóricos diferenciados, os arquivos de autoridade de assunto, quando comparados sob a ótica de seus relacionamentos com os catálogos e com os registros bibliográficos, mostramse semelhantes aos arquivos de autoridade desenvolvidos para pessoas, entidades coletivas e títulos.

\section{Considerações finais}

Após essa revisão de literatura sobre o controle de autoridade nos catálogos em livros e em fichas, nessas considerações finais são traçados alguns apontamentos acerca da primeira parte deste estudo sobre as funções dos dados de autoridade nos catálogos.

A partir do percurso traçado nas seções 3 e 4, onde foram apresentados os tipos de remissivas utilizados por Panizzi e Jewett e as contribuições de Cutter e dos códigos da ALA, é possível notar que o registro de autoridade tal como visto hoje teve sua função e seus elementos (ponto de acesso autorizado, pontos de acesso não autorizados, fontes consultadas, etc.) delineados ainda no contexto dos catálogos analógicos, em especial dos catálogos em fichas, o que ressalta a importância do entendimento acerca desses catálogos para a compreensão dos registros e arquivos de autoridade atuais e do próprio conceito de controle de autoridade.

Nota-se também que as tecnologias utilizadas na criação dos catálogos analógicos tiveram impacto determinante na configuração desses catálogos, de suas possibilidade e limitações e das práticas de catalogação. A possibilidade de acrescentar novas entradas sem prejuízo à ordenação do catálogo, possibilidade essa oferecida pela tecnologia utilizada nos catálogos em fichas, favoreceu a proliferação dos pontos de acesso através dos quais os usuários poderiam encontrar determinado recurso informacional. Com as técnicas de reprodução de fichas, essa possibilidade permitiu que as descrições completas dos recursos informacionais fossem replicadas e apresentadas em diversos locais dentro de um mesmo catálogo, favorecendo, assim, um acesso mais rápido a essas descrições por parte dos usuários.

Além desses exemplos de possibilidades, têm-se também exemplos de limitações decorrentes das tecnologias utilizados na criação dos catálogos. Nos catálogos em fichas, por exemplo, em que as descrições de cada edição ou versão de um recurso informacional eram apresentadas em unidades (fichas) individuais, as diferentes edições e versões de uma mesma obra poderiam estar dispersas ao longo do catálogo e não explicitamente relacionadas, como poderia ocorrer nos catálogos em livros.

Ressalta-se também que as regras de catalogação utilizadas hoje no Brasil, o Anglo-American Cataloguing Rules, 2nd edition, 2002 revision (AACR2r), advêm do contexto anglo-americano em que se desenvolveram os catálogos em livros e em fichas a partir de contribuições como as de Panizzi, Jewett e Cutter. A própria criação do AACR, mesmo tendo ocorrido concomitante ao início da automação das bibliotecas, deu-se no contexto dos catálogos em fichas, de modo que a estrutura desse código, ainda hoje, reflete esse tipo de catálogo. Assim, o estudo sobre os catálogos em fichas apresentado neste trabalho contribui também para a compreensão desse instrumento de representação (o AACR2r).

Por fim, entende-se que a compreensão do controle de autoridade, dos registros de autoridade atuais e do AACR2r são de grande importância no momento atual da Catalogação, em que são discutidos novos conjuntos de regras de descrição, novos padrões para o intercâmbio de dados e novas práticas e tecnologias para a publicação de dados bibliográficos e de autoridade. Com o término deste artigo sobre o controle de autoridade nos catálogos em livros e em fichas, encaminha-se agora a próxima parte deste estudo, que se volta ao controle de autoridade nos catálogos digitais. 


\section{Referências}

ALVES, R. C. V.; SANTOS, P. V. A. da C. Metadados no domínio bibliográfico. Rio de Janeiro: Intertexto, 2013.

AMERICAN LIBRARY ASSOCIATION. A.L.A. Catalog Rules: Author and Title Entries. Chicago: American Library Association, 1941. Disponível em: <http://hdl.handle.net/2027/mdp.39015033890123>. Acesso em: 30 mar. 2016.

ASSUMPÇÃO, F. S.; SANTOS, P. V. A. da C. A importância do controle de autoridade: uma abordagem baseada nos objetivos e nas funções dos catálogos. In: ENCONTRO NACIONAL DE CATALOGADORES, 1., 2012, Rio de Janeiro. Anais... Rio de Janeiro: Fundação Biblioteca Nacional, 2012. Disponível em: <http://hdl.handle.net/10760/18843>. Acesso em: 30 mar. 2016.

AULD, L. Authority Control: An Eighty-Year Review. Library Resources \& Technical Services, v. 26, n. 4, p. 319-330, Oct./Dec. 1982. Disponível em: <http://downloads.alcts.ala.org//rts/lrtsv26no4.pdf>. Acesso em: 30 mar. 2016.

BARBOSA, A. P. Novos rumos da catalogação. Rio de Janeiro: BNG/BRASILART, 1978.

BETTENCOURT, A. M. A representação da informação na Biblioteca Nacional: do documento tradicional ao digital. Rio de Janeiro: Fundação Biblioteca Nacional, 2014. Disponível em: <https://www.bn.br/producao/publicacoes/representacaoinformacao-biblioteca-nacional-documento>. Acesso em: 30 mar. 2016.

BIAGETTI, M. T. Catalogo alfabetico-classificato e catalogo dizionario: le considerazioni e le realizzazioni di Charles A. Cutter. In: NUOVO, A.; PETRUCCIANI, A.; RUFFINI, G. (Org.). Pensare le biblioteche: studi e interventi offerti a Paolo Traniello. Roma: Sinnos, 2008. p. 131-144.

BROWN UNIVERSITY. Catalogue of the Library of Brown University in Providence, Rhode-Island: with an Index of Subjects. Providence: Brown University, 1843. Disponível em: <https://archive.org/details/acataloguelibra12librgoog >. Acesso em: 30 mar. 2016.

BURGER, R. H. Authority Work: the Creation, Use, Maintenance, and Evaluation of Authority Records and Files. Littleton: Libraries Unlimited, 1985.

CALHOUN, K. A Bird's Eye View of Authority Control in Cataloging. In: WORKSHOP ON THE COMPILATION, MAINTENANCE, AND DISSEMINATION OF TAXONOMIC AUTHORITY FILES (TAF), 1998, Washington, DC. Proceedings... [S.I.: s.n.], 1998. Disponível em: <http://researcharchive.calacademy.org/research/informatics/taf/proceedings/Calhoun. html>. Acesso em: 30 mar. 2016

CHAN, L. M. Cataloging and Classification: an Introduction. 2nd ed. New York: McGraw-Hill, 1994.

CHAN, L. M. Cataloging and Classification: an Introduction. 3rd ed. Lanham: Scarecrow Press, 2007.

CINTRA, A. M. M. et al. Para entender as linguagens documentárias. São Paulo: Polis/APB, 1994

CLACK, D. H. Authority Control and Linked Bibliographic Databases. Cataloging \& Classification Quarterly, v. 8, n. 3/4, p. $35-46,1988$

CÓDIGO de catalogação anglo-americano. 2. ed., rev. 2002. São Paulo: FEBAB, 2004.

CUTTER, C. A. Rules for a Dictionary Catalog. 4th ed. rew. Washington, DC: Government Printing Office, 1904

DODEBEI, V. L. D. Tesauro: linguagem de representação da memória documentária. Niterói: Intertexto, 2014.

FIUZA, M. M. Funções e desenvolvimento do catálogo: uma visão retrospectiva. Revista da Escola de Biblioteconomia da UFMG, v. 9, n. 2, p. 139-158, set. 1980

FRÍAS, J. A.; PÉREZ ALVAREZ, M. I. La ordenación de los asientos bibliográficos en los catálogos: hacia una normativa internacional. Revista Española de Documentación Científica, v. 18, n. 3, p. 283-297, 1995. Disponível em: <http://dx.doi.org/10.3989/redc.1995.v18.i3.645>. Acesso em: 30 mar. 2016.

GARRIDO ARILLA, M. R. Teoría e historia de la catalogación de documentos. Madrid: Síntesis, 1996.

GUERRINI, M. Verso una nuova struttura catalografica dei dati bibliografici. In: ROSSI, F.; TINTI, P. (Org.). Belle contrade della memoria: studi su documenti e libri in onore di Maria Gioia Tavoni. Bologna: Patron, 2009. Disponível em: $<$ http://hdl.handle.net/10760/13364>. Acesso em: 07 mar. 2016.

INTERNATIONAL FEDERATION OF LIBRARY ASSOCIATIONS AND INSTITUTIONS. Statement of International Cataloguing Principles. [S.I.]: IFLA, 2009. Disponível em: <http://www.ifla.org/files/cataloguing/icp/icp 2009-en.pdf>. Acesso em: 30 mar. 2016

JEWETT, C. C. Preface. In: BROWN UNIVERSITY. Catalogue of the Library of Brown University in Providence, RhodeIsland: with an Index of Subjects. Providence: Brown University, 1843. p. iii-xxi. Disponível em: $<$ https://archive.org/details/acataloguelibra12librgoog >. Acesso em: 30 mar. 2016. 
LUBETZKY, S. Crisis in the Catalog. In: SVENONIUS, E.; MCGARRY, D. (Eds.). Seymour Lubetzky: Writings on the Classical Art of Cataloging. Englewood: Libraries Unlimited, 2001. Paper read before the Large Libraries Round Table of the Catalog Section, ALA, San Francisco, June 20, 1939. Originalmente publicado em: Cataloguers' and Classifiers' Yearbook 8, p. 48-54, 1940.

LUBETZKY, S. The Vicissitudes of Ideology and Technology in Anglo-American Cataloging since Panizzi and a Prospective Reformation of the Catalog for the Next Century. In: SVENONIUS, E.; MCGARRY, D. (Eds.). Seymour Lubetzky: Writings on the Classical Art of Cataloging. Englewood: Libraries Unlimited, 2001. Originalmente publicado em: The future of cataloging: insights from the Lubetzky Symposium, April 18, 1998. Chicago: American Library Association, 2000.

MARAIS, $\mathrm{H}$. Authority control in an academic library consortium using a union catalogue maintained by a central office for authority control. 2004. $310 \mathrm{f}$. Tese (Doctor of literature and philosophy in the subject Information Science) - University of South Africa, Petroria, 2004. Disponível em: <http://uir.unisa.ac.za/handle/10500/2546>. Acesso em: 30 mar. 2016.

MAXWELL, R. L. Maxwell's Guide to Authority Work. Chicago: American Library Association, 2002.

MEY, E. S. A.; SILVEIRA, N. C. Catalogação no plural. Brasília: Briquet de Lemos/Livros, 2009.

OBILLE, K. L. B. AACR2 to RDA: a Paradigm Shift? Journal of Philippine Librarianship, v. 32, p. 1-12, 2012. Disponível em: $<$ http://journals.upd.edu.ph/index.php/jpl/article/view/3616>. Acesso em: 30 mar. 2016.

PANIZZI, A. Rules for the Compilation of the Catalogue. In: BRITISH MUSEUM. Catalogue of Printed Books in the British Museum: Volume I. London: J. B. Nichols and Son Printers, 1841. p. v-ix. Disponível em: <http://hdl.handle.net/2027/umn.31951001998306w>. Acesso em: 30 mar. 2016.

SANTOS, P. L. V. A. da C.; PEREIRA, A. M. Catalogação: breve histórico e contemporaneidade. Niterói: Intertexto, 2014.

SAYERS, W. C. B.; STEWART, J. D. The Card Catalogue: a Practical Manual For Public and Private Libraries: including other uses of cards in libraries. London: Grafton \& Company, 1913. Disponível em:

$<$ https://archive.org/details/cardcataloguepra00sayerich>. Acesso em: 30 mar. 2016.

STROMGREN, P. Charles Ammi Cutter: Library Systematizer Extraordinaire. Northampton: Forbes Library, 2004. Disponível em: <http://forbeslibrary.org/info/library-history/charles-ammi-cutter/>. Acesso em: 30 mar. 2016.

SVENONIUS, E. The Intellectual Foundation of Information Organization. Cambridge: MIT Press, 2000.

TAYLOR, A. G. Authority files in online catalogs: an investigation of their value. Cataloging \& Classification Quarterly, v. 4, n. 3, p. 1-17, 1984.

TAYLOR, A. G. Introduction to Cataloging and Classification. 10th ed. Westport: Libraries Unlimited, 2006.

TAYLOR, A. G.; JOUDREY, D. N. The Organization of Information. 3rd ed. Westport: Libraries Unlimited, 2009.

TILLETT, B. B. Bibliographic Structures: the Evolution of Catalog Entries, References, and Tracings. In: SVENONIUS, E. (Org.). Conceptual Foundations of Descriptive Cataloging. San Diego: Academic Press, 1989, p. 149-165.

TILLETT, B. B. The History of Linking Devices. Library Resources and Technical Services, v. 36, n. 1, p. 23-36, Jan. 1992. 


\section{Dados dos autores}

\section{Fabrício Silva Assumpção}

Bacharel em Biblioteconomia pela Universidade Estadual Paulista "Júlio de Mesquita Filho" (UNESP), Campus de Marília (2011), mestre em Ciência da Informação pela UNESP (2013) e doutorando em Ciência da Informação pela UNESP (2014-). Bibliotecário na Universidade Federal do Paraná (UFPR), Setor Litoral. assumpcao.f@gmail.com

\section{Plácida Leopoldina Ventura Amorim da Costa Santos}

Professora na Universidade Estadual Paulista "Júlio de Mesquita Filho" (UNESP), Campus de Marília. Programa de Pós-Graduação em Ciência da Informação.placidasantos@gmail.com

\section{Zaira Regina Zafalon}

Professora na Universidade Federal de São Carlos (UFSCar). Programa de Pós-Graduação em Ciência da Informação (UFSCar). zaira@ufscar.br

Recebido - Received: 2016-07-25

Aceitado - Accepted: 2016-10-19

\footnotetext{
1 Uma entidade é entendida aqui como "Algo com caráter unitário e autocontido; algo com existência independente ou separada; uma abstração, um conceito ideal, um objeto de pensamento ou um objeto transcendental" (INTERNATIONAL FEDERATION OF LIBRARY ASSOCIATIONS AND INSTITUTIONS, 2009, p. 11, tradução nossa).

2 "Cabeçalho" é sinônimo de ponto de acesso. Essa denominação advém dos catálogos em fichas, em que os pontos de acesso eram incluídos no início da ficha catalográfica, como um cabeçalho de uma página.

${ }^{3}$ Atualmente, nos catálogos digitais, o termo "entrada" tem sido utilizado como sinônimo de "ponto de acesso". No entanto, nos catálogos analógicos, tal termo é utilizado para se referir a um registro bibliográfico, como é explicitado no Glossário do AACR2r: "Entrada. Entry. Registro de um item no catálogo." (CÓDIGO..., 2004, p. D5).

${ }^{4}$ Cabe aqui uma menção à nota feita por Svenonius (2000, p. 208, tradução nossa): "Às vezes, várias fichas eram utilizadas na descrição de um documento e, ocasionalmente, fichas com entradas tracejadas eram utilizadas para descrever vários documentos".
}

\section{$(\mathrm{cc}) \mathrm{BY}$}

This work is licensed under a Creative Commons Attribution 4.0

United States License.

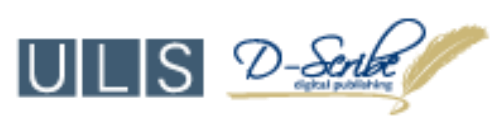

This journal is published by the University Library System of the University of Pittsburgh as part of its D-Scribe Digital Publishing Program and is cosponsored by the University of Pittsburgh Press. 